\begin{tabular}{|c|l|}
\hline Title & ATP content and maturational /developmental ability of bovine oocytes with various cytoplasmic morphologies \\
\hline Author(s) & Nagano, Masashi; Katagiri, Seiji; Takahashi, Y oshiyuki \\
\hline Citation & $\begin{array}{l}\text { Zygote, 14(4), 299-304 } \\
\text { https://doi.org/40.1017/S0967199406003807 }\end{array}$ \\
\hline Issue Date & 2006-11 \\
\hline Doc URL & http://hdl.handle.net/2115/17148 \\
\hline Rights & Copyright $\odot$ 2006 Cambridge University Press \\
\hline Type & article \\
\hline File Information & ZYGOTE14 4.pdf \\
\hline
\end{tabular}

Instructions for use 


\title{
ATP content and maturational/developmental ability of bovine oocytes with various cytoplasmic morphologies
}

\author{
Masashi Nagano, Seiji Katagiri and Yoshiyuki Takahashi \\ Laboratory of Theriogenology, Department of Veterinary Clinical Sciences, Graduate School of Veterinary Medicine, \\ Hokkaido University, Sapporo 060-0818, Japan
}

Date submitted: 09.11.05. Date accepted: 01.02.06

\section{Summary}

\begin{abstract}
We examined the relationship among morphological appearance (six groups) of bovine oocytes, ATP content and maturational/developmental ability. Oocytes with a brown ooplasm (with or without a dark region) had intermediate levels of ATP at the germinal vesicle (GV) stage and showed higher rates of first polar body (PB) extrusion than the other groups. Oocytes with a low level of ATP (oocytes with a pale ooplasm without dark clusters) and oocytes with a high level of ATP (oocytes with a black ooplasm) showed lower rates of PB extrusion. During in vitro maturation, ATP levels in oocytes decreased at around GV breakdown and increased toward metaphase II (MII). MII oocytes having a brown ooplasm with a dark region, which had good developmental capacity, had a relatively high level of ATP. MII oocytes with a brown or pale ooplasm without dark clusters, which had poor developmental capacity, had low ATP levels. MII oocytes with a black ooplasm, which had poor developmental capacity, had an unusually high level of ATP. These results suggest that the morphological appearance of bovine oocytes is closely related to their ATP levels and that cytoplasmic morphology will give an advantage for the selection of oocytes with a high maturational and developmental ability.
\end{abstract}

Keywords: ATP, Developmental ability, Maturation, Mitochondria, Oocyte morphology

\section{Introduction}

In a previous study (Nagano et al., 2006), we classified oocytes from slaughterhouse-derived ovaries into seven groups by their morphological appearance and examined the relationship between the developmental potential of oocytes and their morphology before in vitro maturation (IVM). It was found that the oocytes with a brown ooplasm had excellent developmental capacity, and that the oocytes with dark clusters had a large number of lipid droplets in their ooplasm and the greatest developmental capacity after in vitro fertilization (IVF). We also found that lipid droplets were located in association with mitochondria and the smooth endoplasmic reticulum (Nagano et al., 2006). The association may indicate the utilization of lipids

All correspondence to: Y. Takahashi, Laboratory of Theriogenology, Department of Veterinary Clinical Sciences, Graduate School of Veterinary Medicine, Hokkaido University, Kita-ku Kita 18 Nishi 9, Sapporo 060-0818, Japan. Tel: +81 11706 5231. Fax: +81 11706 5232. e-mail: ytaka@vetmed.hokudai.ac.jp as an energy substrate for the synthesis of adenosine triphosphate (ATP) by mitochondria. It was reported that bovine oocytes use lipids as an oxidative substrate for nuclear and/or cytoplasmic maturation (Cetica et al., 2002; Kim et al., 2001; Rieger \& Loskutoff, 1994). Thus, the storage of lipid droplets in the ooplasm seems to be important for the production of ATP and maturational ability of oocytes. From this result, it is predicted that oocytes with a large amount of lipid droplets have high levels of ATP and good developmental capacity. However, oocytes with a black ooplasm had less developmental capacity, although they have an abundance of lipid droplets in their ooplasm (Nagano et al., 2006).

A previous report showed that bovine oocytes with multi-layered cumulus cells had higher levels of ATP before and after IVM and greater potential to develop to the blastocyst stage than denuded oocytes (Stojkovic et al., 2001). However, the lower ATP levels of denuded oocytes can be attributed to a lack of ATP supplied from the cumulus cells. In humans, the potential for embryonic development after IVF and implantation after transfer was associated with embryos developed 
from cohorts of in vivo matured oocytes with higher levels of ATP (Van Blerkom et al., 1995). However, no study has compared ATP content among oocytes at the germinal vesicle $(\mathrm{GV})$ stage with a multi-layered cumulus investment and showing various features of the ooplasm or examined the changes in ATP content during the IVM culture of bovine oocytes. During nuclear and cytoplasmic maturation, a rearrangement of organelles occurs. Notably, the organization and metabolic activity of mitochondria (ATP production) seem to be correlated with cytoplasmic maturation and a resumption of meiosis (Calarco, 1995; Hyttel et al., 1986; Kruip et al., 1983; Van Blerkom \& Runner, 1984). Therefore, the relationship between ATP content and the maturational and developmental capacity of oocytes must be clarified.

In the present study, we examined the relationship between the nuclear maturational ability of oocytes varying in ooplasmic appearance and the ATP content of GV oocytes (before IVM), and also investigated the change in the ATP level during IVM. Finally, we discuss the relationship between the ATP level and developmental ability of oocytes at metaphase II (MII) using our previous results (Nagano et al., 2006).

\section{Materials and methods}

\section{Recovery of oocytes}

Bovine ovaries obtained at a local abattoir were kept in normal saline at $25-30{ }^{\circ} \mathrm{C}$ and delivered to the laboratory within $6 \mathrm{~h}$ of their collection. Cumulus-oocyte complexes (COCs) were collected from small antral follicles $(2-8 \mathrm{~mm}$ in diameter) by aspiration as described previously (Nagano et al., 1999). All COCs were washed twice in HEPES-buffered Tyrode's medium (TALP-HEPES) (Bavister et al., 1983) supplemented with $3 \mathrm{mg} / \mathrm{ml}$ of bovine serum albumin (BSA, fraction $\mathrm{V}$, Sigma), $0.2 \mathrm{mM}$ sodium pyruvate and $50 \mu \mathrm{g} / \mathrm{ml}$ of gentamicin sulfate (Sigma). During the washing procedure, oocytes were examined under a stereomicroscope and classified into six groups according to cytoplasmic appearance as shown in Table 1 (Nagano et al., 2006). The oocytes with a small ooplasm $(<115 \mu \mathrm{m}$ in diameter, group 7 in the previous study; Nagano et al., 2006) were excluded in the present study since these small oocytes are in the growing phase and, thus, have not acquired developmental capacity.

\section{IVM of COCs}

The COCs were subjected to IVM as described previously (Takahashi et al., 1996). Briefly, the COCs were washed once in a maturation medium consisting of 25 mM HEPES-buffered TCM 199 (Gibco
Table 1 Cytoplasmic appearance of oocytes in the six groups

\begin{tabular}{lll}
\hline & \multicolumn{2}{c}{ Cytoplasmic characteristics } \\
\cline { 2 - 3 } Oocyte group & Colour & \multicolumn{1}{c}{ Appearance } \\
\hline 1 & Brown & Homogeneous \\
2 & Brown & $\begin{array}{c}\text { Homogeneous with a dark zone } \\
\text { around the periphery }\end{array}$ \\
3 & Brown & $\begin{array}{l}\text { Heterogeneous with dark clusters } \\
4\end{array}$ \\
5 & Pale & Homogeneous \\
6 & Pale & Heterogeneous with dark clusters \\
\hline
\end{tabular}

All oocytes had several layers of unexpanded cumulus cells. Oocytes of groups $1-6$ were $\geq 115 \mu \mathrm{m}$ in diameter.

Laboratories) supplemented with $10 \%$ fetal calf serum (FCS, Gibco), 0.02 units $/ \mathrm{ml}$ of follicle stimulating hormone (FSH, Sigma), $1 \mu \mathrm{g} / \mathrm{ml}$ of estradiol$17 \beta$ (Sigma), $0.2 \mathrm{mM}$ sodium pyruvate and $50 \mu \mathrm{g} / \mathrm{ml}$ of gentamicin sulfate. They were then cultured in $50 \mu \mathrm{l}$ droplets of maturation medium (about 10 COCs per droplet) covered with paraffin oil at $39^{\circ} \mathrm{C}$ in a humidified atmosphere of $5 \% \mathrm{CO}_{2}$ in air. After various culturing periods ( 3 to $22 \mathrm{~h}$ ) for IVM, COCs were transferred to $0.5 \mathrm{ml}$ of TALP-HEPES in a $15 \mathrm{ml}$ conical tube, and cumulus cells surrounding oocytes were removed by vortexing for $4 \mathrm{~min}$. If the cumulus cells remained around oocytes, they were removed using a fine glass pipette.

\section{Evaluation of the nuclear status of oocytes}

Denuded oocytes were fixed with ethanol:acetic acid (3:1) and stained with a 1\% aceto-orcein solution. Their nuclear status was examined under a phase-contrast microscope.

\section{Measurement of ATP content in individual oocytes}

The ATP content of individual oocytes was measured according to a previous report (Rieger, 1997). Briefly, a denuded oocyte was washed four times in the sample buffer and transferred to $25 \mu \mathrm{l}$ of the sample buffer in a $1.5 \mathrm{ml}$ tube. The sample buffer consisted of $99.0 \mathrm{mM}$ $\mathrm{NaCl}, 3.1 \mathrm{mM} \mathrm{KCl}, 0.35 \mathrm{mM} \quad \mathrm{NaH}_{2} \mathrm{PO}_{4}, 21.6 \mathrm{mM}$ sodium lactate, $10.0 \mathrm{mM}$ HEPES, $2.0 \mathrm{mM} \mathrm{CaCl}_{2}, 1.1 \mathrm{mM}$ $\mathrm{MgCl}_{2}, 25.0 \mathrm{mM} \mathrm{NaHCO}, 1.0 \mathrm{mM}$ sodium pyruvate, $0.1 \mathrm{mg} / \mathrm{ml}$ of gentamicin sulfate and $6.3 \mathrm{mg} / \mathrm{ml}$ of BSA (Rieger, 1997). These tubes were placed in boiling water for $3 \mathrm{~min}$ to inactivate the endogenous phosphatases and then frozen at $-80^{\circ} \mathrm{C}$ until assayed. All assay reagents (assay mix, assay mix dilution buffer, ATP stock solution and somatic cell releasing agent) were purchased as a kit (ATP bioluminescent somatic cell assay kit, FL-ASC, Sigma) and prepared according to the manufacturer's instructions. Aliquots of $100 \mu \mathrm{g} / \mathrm{ml}$ 
of ATP stock solution and the assay mix were thawed just before use. The ATP stock solution was diluted to concentrations of 2 to $20 \mathrm{pmol} / 25 \mu \mathrm{l}$ in sample buffer. The assay mix was diluted 1:25 in assay mix dilution buffer. The ATP standards and samples in $1.5 \mathrm{ml}$ tubes were kept on crushed ice, and $50 \mu$ of ice-cold somatic cell-releasing agent was added to all tubes. The tubes were kept on ice for $5 \mathrm{~min}$ before the addition of $100 \mu \mathrm{l}$ of ice-cold 1:25 assay mix. After that the contents of the tubes were transferred to a white 96-well plate (Labsystems). Then, $100 \mu \mathrm{l}$ of assay mix was added to each well at $5 \mathrm{~s}$ intervals and held at room temperature for $5 \mathrm{~min}$ to pass through the initial chemiluminescence flash period. ATP content was quantified by measuring the luminescence (Luminesensor JNR AB-2100).

\section{Staining of active mitochondria}

Denuded oocytes were stained with rhodamine 123 (Molecular Probes) as described previously (Barnett et al., 1996) with slight modifications. Briefly, oocytes were incubated in TCM-199 with $10 \mu \mathrm{g} / \mathrm{ml}$ of rhodamine 123 for $15 \mathrm{~min}$ at $39^{\circ} \mathrm{C}$ in a humidified atmosphere of $5 \% \mathrm{CO}_{2}$ in air. Stained oocytes were washed twice in Dulbecco's phosphate-buffered saline (DPBS) and transferred to $10 \mu \mathrm{l}$ of DPBS in a Terasaki plate (Sumitomo Bakelite). Confocal images were obtained using a confocal laser scanning microscope (FluoView FV500, Olympus) equipped with a heliumneon mixed-gas laser operated at $543 \mathrm{~nm}$ excitation and a fluorescein filter.

\section{Experimental design}

\section{Experiment 1}

The relationship between the maturational ability and ATP content of oocytes was examined. Oocytes at the GV stage were collected and classified into six groups (Table 1). Some oocytes in each group were used to examine ATP content immediately after their collection from the ovaries while others were subjected to IVM culture $(22 \mathrm{~h})$ and used to examine the extrusion rate of the first PB as a marker of their maturational ability. Then, oocytes with a clearly distinguishable PB were subjected to measurements of ATP content.

\section{Experiment 2}

To examine the changes in ATP content during IVM in the oocytes with maturational competence, the oocytes in groups 1 and 2 (most of the oocytes which extruded a PB in experiment 1 ) were cultured for IVM. The ATP content and the nuclear status of oocytes in each group were examined at $0,3,6,9,12,17$ and $22 \mathrm{~h}$ after IVM culture.

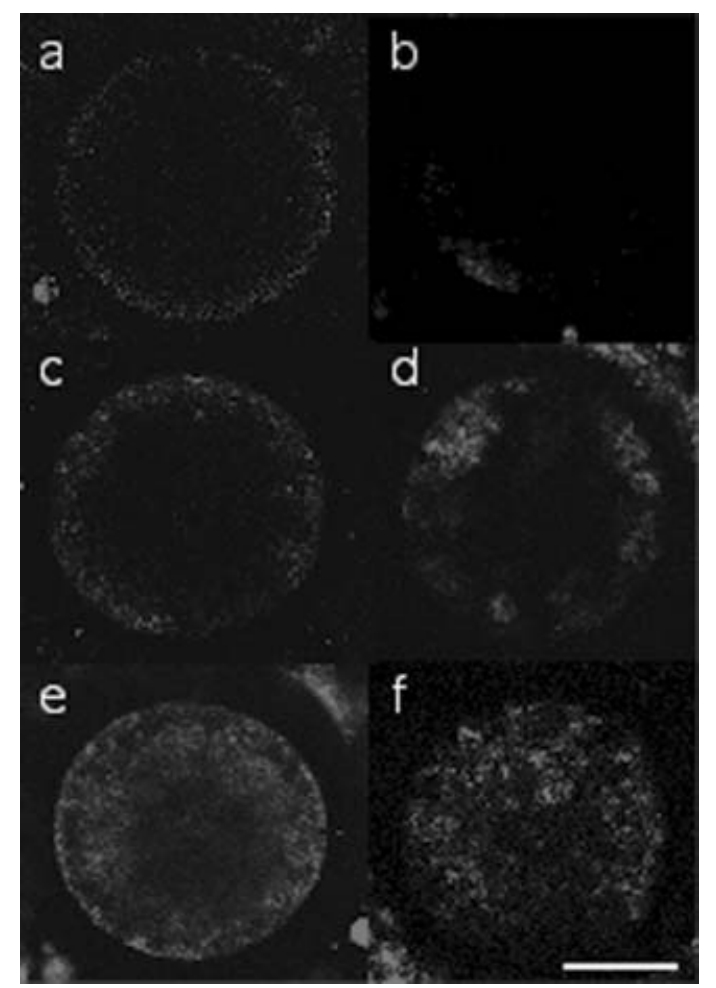

Figure 1 Confocal images of active mitochondria. The active mitochondria were stained with rhodamine 123 and confocal images were obtained using a confocal laserscanning microscope equipped with a helium-neon mixedgas laser operated at $543 \mathrm{~nm}$ excitation and a fluorescein filter. The staining pattern was classified into three types: type 1 $(a, b)$ mitochondria were stained weakly and detected at the periphery of the ooplasm; type $3(e, f)$ mitochondria were stained strongly and distributed throughout the ooplasm; and type $2(c, d)$ the staining was intermediate in strength and distribution between types 1 and 3 . Bar $=50 \mu \mathrm{m}$.

\section{Experiment 3}

The relationship between oocyte morphology and the distribution of active mitochondria was examined. Oocytes in each group were stained with rhodamine 123 before and after IVM culture $(22 \mathrm{~h})$. The pattern of the mitochondrial distribution in the ooplasm was classified into three types: type 1 , mitochondria were stained weakly and detected at the periphery of the ooplasm (Fig. 1a, b); type 3, mitochondria were stained strongly and distributed throughout the ooplasm (Fig. 1e, f); and type 2 (Fig. 1c, d), the staining was intermediate in strength and distribution between types 1 and 3 .

\section{Statistical analysis}

The differences in ATP content among oocyte groups and the changes in ATP content during IVM culture at different time points were analysed using a oneway analysis of variance followed by Tukey-Kramer's 
Table 2 The ATP content of oocytes before and after IVM

\begin{tabular}{llll}
\hline Oocyte & $\begin{array}{l}\text { ATP content (pmol/oocyte) of oocyte } \\
\text { group }\end{array}$ & $\begin{array}{l}\text { B of PB } \\
\text { extrusion } \\
\text { after IVM } \\
(n=21)\end{array}$ \\
\hline 1 & $0.61 \pm 0.35^{a b c}$ & $0.81 \pm 0.23^{c}$ & $76.2^{a}$ \\
& $(18)$ & $(16)$ & \\
2 & $0.66 \pm 0.30^{a b c}$ & $0.88 \pm 0.17^{b c}$ & $81.0^{a}$ \\
3 & $(18)$ & $(17)$ & \\
& $0.53 \pm 0.20^{b c}$ & $1.00 \pm 0.26^{b c}$ & $85.7^{a}$ \\
4 & $(18)$ & $(18)$ & \\
& $0.47 \pm 0.37^{c}$ & $0.70 \pm 0.25^{c}$ & $28.6^{c}$ \\
5 & $(18)$ & $(6)$ & \\
& $0.76 \pm 0.47^{a b}$ & $1.18 \pm 0.35^{a b}$ & $66.7^{a b}$ \\
6 & $(18)$ & $(14)$ & \\
& $0.95 \pm 0.49^{a}$ & $1.39 \pm 0.58^{a}$ & $42.9^{b c}$ \\
& $(18)$ & $(9)$ & \\
\hline
\end{tabular}

Values are the mean \pm SD. The numbers in parentheses are the number of oocytes whose ATP contents were measured. *ATP content of oocytes with a first polar body (PB).

${ }^{a-c}$ Different superscripts indicate a significant difference in the same column $(p<0.05)$.

Honestly Significant Difference as a post-hoc test using software (JMP version 5, SAS Institute). The ATP content before and after IVM in each group was compared with Student's $t$-test. The extrusion of a PB was analysed using Fisher's exact test. The mitochondrial distribution pattern before and after IVM in each group was compared with the chi-square test. These analyses were performed using software (StatView version 4.5, Abacus Concepts).

\section{Results}

\section{Experiment 1}

The ATP content and rates of PB extrusion of oocytes are shown in Table 2 . The oocytes in groups 1-3 showed the highest PB extrusion rate $(p<0.05)$, and had intermediate levels of ATP before IVM culture (at the GV stage). The PB extrusion rate and ATP levels of GV oocytes in group 4 were the lowest $(p<0.05)$. Group 6 had a lower PB extrusion rate than groups 1-3 but the highest levels of ATP at the GV stage. The PB extrusion rate and ATP level of group 5 were between those of groups 1-3 and 6 .

After IVM, the ATP levels of oocytes with a PB (MII oocytes) were higher than those of GV oocytes in all groups $(p<0.05)$. MII oocytes in groups $2-3$ had intermediate levels of ATP. The ATP levels of MII oocytes were highest in group 6 and lowest in groups 1 and 4 . The ATP levels of MII oocytes in group 5 were between those in groups 2-3 and 6 .


Figure 2 Nuclear status and ATP content in group 1 and 2 oocytes during in vitro maturational culture (IVM). (a) Transition of nuclear status during IVM $(n=20$ each). (b) Change of ATP content ( $n=20$ each). Different superscripts $(\mathrm{a}-\mathrm{c})$ mean significant differences $(p<0.05)$. AI/TI, anaphase I/telophase I; CC, chromosomal condensation; GV, germinal vesicle; GVBD, germinal vesicle breakdown; $\mathrm{MI}$, metaphase I; MII, metaphase II.

\section{Experiment 2}

Changes of ATP content and nuclear status during IVM culture are shown in Fig. 2. Most of the oocytes were at the GV stage at 0 and $3 \mathrm{~h}$ after IVM. At $6 \mathrm{~h}$ after IVM, $65 \%$ of oocytes were beyond the GV breakdown (GVBD) stage, and the ATP level was lowest. At $9 \mathrm{~h}$ after IVM, 95\% of oocytes had formed chromosomes (chromosomal condensation and metaphase I (MI)), and at $12 \mathrm{~h}$, most had reached the MI stage. During this period, the ATP content was increasing. At $17 \mathrm{~h}$ after IVM, about $80 \%$ of oocytes were at the anaphase I to MII stage and the ATP content reached a maximal level.

\section{Experiment 3}

As shown in Table 3, the distribution of active mitochondria did not change before and after IVM, except in group $6(p<0.05)$. The majority of oocytes in groups 1-3 and 5 exhibited a staining pattern of type 2 or 3 before and after IVM. Four out of 10 oocytes in group 4 exhibited a type 1 pattern after IVM. The staining pattern in group 6 was mainly type 1 before IVM, and mainly types 2 and 3 after IVM. After IVM, all oocytes 
Table 3 The number of oocytes with each type of mitochondrial distribution

\begin{tabular}{|c|c|c|c|c|c|c|c|}
\hline \multirow{2}{*}{$\begin{array}{l}\text { Oocyte } \\
\text { group }\end{array}$} & \multicolumn{3}{|c|}{ Before IVM $(n=10)$} & \multicolumn{3}{|c|}{ After IVM $(n=10)$} & \multirow{2}{*}{$\begin{array}{c}\text { No. of oocytes } \\
\text { with PB }\end{array}$} \\
\hline & Type 1 & Type 2 & Type 3 & Type 1 & Type 2 & Type 3 & \\
\hline 1 & 0 & 5 & 5 & 0 & 6 & 4 & 7 \\
\hline 2 & 1 & 5 & 4 & 2 & 4 & 4 & 8 \\
\hline 3 & 0 & 4 & 6 & 0 & 2 & 8 & 10 \\
\hline 4 & 3 & 4 & 3 & 4 & 4 & 2 & 4 \\
\hline 5 & 2 & 2 & 6 & 2 & 4 & 4 & 6 \\
\hline $6^{*}$ & 5 & 4 & 1 & 1 & 4 & 5 & 4 \\
\hline
\end{tabular}

*Distribution pattern differed before and after IVM $(p<0.05)$.

that showed a type 3 staining pattern had $\mathrm{PB}$, except for one oocyte in group 6.

\section{Discussion}

The timing of the nuclear progression of oocytes in groups 1 and 2 under the present IVM conditions was similar to that in a previous report (Sirard et al., 1989). It was reported that the rate of protein synthesis in bovine oocytes was higher at the beginning of IVM culture until the appearance of MI and then decreased (Coenen et al., 2004). Oocytes may use a larger amount of ATP for production of proteins and these proteins may be necessary for the progression of GVBD. After the GVBD stage, the ATP content of oocytes increased in the present study. The junction between cumulus cell projections and the ooplasm was disrupted concomitantly with GVBD (Hyttel et al., 1986). Therefore, oocytes may not be able to obtain the ATP produced by cumulus cells after the GVBD stage. A previous study reported that bovine oocytes showed an increase in lipase activity after IVM culture compared with before IVM culture (Cetica et al., 2002). Therefore, the increase in ATP content after GVBD in oocytes may reflect an increase in the production of ATP using lipids.

Few lipid droplets were observed in the ooplasm in group 4 before and after IVM (Nagano et al., 2006). It seems that GV oocytes with a pale ooplasm (group 4) have low levels of energy substrate (lipid droplets) and, thus, can not produce the ATP required to complete nuclear maturation. GV oocytes having a pale ooplasm with dark clusters (group 5) and those with a black ooplasm (group 6) had higher levels of ATP but showed lower rates of PB extrusion than the oocytes with a brown ooplasm. The GV oocytes in groups 5 and 6 had a similar arrangement of organelles, including the distribution of mitochondria, to that of matured oocytes, and accumulated lipid droplets in their ooplasm (Nagano et al., 2006). Thus, GV oocytes in groups 5 and 6 may have produced a larger amount of ATP before they were collected from ovarian follicles, or unusually high levels of ATP in ooplasm may indicate a disruption of oocyte functions for consuming ATP.

In the present study, the ATP levels of MII oocytes were higher than those of GV oocytes in all groups, and the trend of PB extrusion (nuclear maturation) between oocyte groups was comparable to that of normal fertilization and cleavage rates of corresponding oocyte groups in the previous study (Nagano et al., 2006). MII oocytes in groups 2, 3 and 5 had moderate levels of ATP. In a previous study (Nagano et al., 2006), the cleaved oocytes in groups 2, 3 and 5 showed high rates of development to the blastocyst stage $(24.4 \%, 29.4 \%$ and $24.0 \%$, respectively). Although the PB extrusion rate in this study and the cleavage rate $(54.6 \%)$ in the previous study were low in group 5 , the rate at which cleaved embryos developed into blastocysts in group 5 was higher and similar to that in groups 23 in the previous study (Nagano et al., 2006). Further, oocytes in groups 1 and 4, which showed small cell numbers in resulting blastocysts (119.9 and 98.5 cells per blastocyst, respectively) compared with those in groups 2, 3 and 5 (158.8, 178.8 and 184.6 cells per blastocyst, respectively) and low rates of development to the blastocyst stage (group 4, 9.2\%) (Nagano et al., 2006), had less ATP, possibly due to fewer active mitochondria. ATP is mainly used by microtubules and actin filaments during cell division (Wanka \& Van Zoelen, 2003). Thus, MII oocytes with low levels of ATP may develop into blastocysts with a small number of cells. MII oocytes in group 6 had the highest levels of ATP, but a low rate of development into blastocysts (7.5\%; Nagano et al., 2006). These results clearly indicate that storage of ATP at proper levels in MII oocytes is one of the key factors determining subsequent embryonic development and the quality of resulting blastocysts. More importantly, the results suggest that excessively high levels of ATP in matured oocytes may indicate an impaired developmental capacity and disruption of the control of mitochondrial functions of oocytes. A careful investigation of the relationship between the 
developmental capacity of oocytes, ATP content and mitochondrial activity is needed for confirmation of the results of the present study.

The present study revealed that oocytes having a brown ooplasm with a dark region at the periphery or dark clusters (having the proper amount of lipid droplets) in their ooplasm produced relatively high levels of ATP at the GV and MII stages, resulting in good maturational and subsequent developmental ability. The oocytes with a pale ooplasm (few lipid droplets) had low levels of ATP at the GV and MII stages, leading to poor maturational and developmental ability. In contrast, oocytes with a black ooplasm (large amounts of lipid droplets) had unusually high levels of ATP at the GV stage, leading to low maturational and subsequent developmental ability. In conclusion, these results suggest that the morphological appearance of bovine oocytes is closely related to their ATP levels and that cytoplasmic morphology provides an indication for the selection of oocytes with a high maturational and developmental ability.

\section{Acknowledgements}

This study was supported by Grants-in-Aid for Scientific Research (Nos. 09660296, 12760183 and 13460136) from the Ministry of Education, Science, Sports and Culture, Japan to Y.T. and M.N. We thank the staff of the Hayakita Meat Inspection Office for the collection of bovine ovaries.

\section{References}

Barnett, D.K., Kimura, J. \& Bavister, B.D. (1996). Translocation of active mitochondria during hamster preimplantation embryo development studies by confocal laser scanning microscopy. Dev. Dyn. 205, 64-72.

Bavister, B.D., Leibfried, M.L. \& Lieberman, G. (1983). Development of preimplantation embryos of the golden hamster in a defined culture medium. Biol. Reprod. 28, 23547.

Calarco, P.G. (1995). Polarization of mitochondria in the unfertilized mouse oocyte. Mol. Reprod. Dev. 16, 36-43.

Cetica, P., Pintos, L., Dalvit, G. \& Beconi, M. (2002). Activity of key enzymes involved in glucose and triglyceride catabolism during bovine oocyte maturation in vitro. Reproduction 124, 675-81.

Coenen, K., Massicotte, L. \& Sirard, M.A. (2004). Study of newly synthesized proteins during bovine oocytes maturation in vitro using image analysis of twodimensional gel electrophoresis. Mol. Reprod. Dev. 67, 31322.

Hyttel, P., Xu, K.P., Smith, S. \& Greve, T. (1986). Ultrastructure of in vitro oocyte maturation in cattle. J. Reprod. Fertil. 78, 615-25.

Kim, J.Y., Kinoshita, M., Ohnishi, M. \& Fukui, Y. (2001). Lipid and fatty acid analysis of fresh and frozen-thawed immature and in vitro matured bovine oocytes. Reproduction 122, 131-8.

Kruip, T.A.M., Cran, D.G., Van Beneden, T.H. \& Dieleman, S.J. (1983). Structural changes in bovine oocytes during final maturation in vivo. Gamete Res. 8, 29-47.

Nagano, M., Takahashi, Y. \& Katagiri, S. (1999). In vitro fertilization and cortical granule distribution of bovine oocytes having heterogeneous ooplasm with dark clusters. J. Vet. Med. Sci. 61, 531-5.

Nagano, M., Katagiri, S. \& Takahashi, Y. (2006). Relationship between bovine oocyte morphology and in vitro developmental potential. Zygote 14, 53-61.

Rieger, D. (1997). Batch analysis of the ATP content of bovine sperm, oocytes, and early embryos using a scintillation counter to measure the chemiluminescence produced by the luciferin-luciferase reaction. Anal. Biochem. 246, 67-70.

Rieger, D. \& Loskutoff, N. (1994). Changes in the metabolism of glucose, pyruvate, glutamine and glycine during maturation of cattle oocytes. J. Reprod. Fertil. 100, 257-62.

Sirard, M.A., Florman, H.M., Leibfried-Rutledge, M.L., Barnes, F.L., Sims, M.L. \& First, N.L. (1989). Timing of nuclear progression and protein synthesis necessary for meiotic maturation of bovine oocytes. Biol. Reprod. 40, 125763.

Stojkovic, M., Machado, S.A., Stojkovic, P., Zakhartchenko, V., Hutzler, P., Goncalves, P.B. \& Wolf, E. (2001). Mitochondrial distribution and adenosine triphosphate content of bovine oocytes before and after in vitro maturation: correlation with morphological criteria and developmental capacity after in vitro fertilization and culture. Biol. Reprod. 64, 9049.

Takahashi, Y., Hishinuma, M., Matsui, M., Tanaka, H. \& Kanagawa, H. (1996). Development of in vitro matured/fertilized bovine embryos in a chemically defined medium: influence of oxygen concentration in the gas atmosphere. J. Vet. Med. Sci. 58, 897-902.

Van Blerkom, J. \& Runner, M.N. (1984). Mitochondrial reorganization during resumption of arrested meiosis in the mouse oocyte. Am. J. Anat. 171, 335-55.

Van Blerkom, J., Davis, P.W. \& Lee, J. (1995). ATP content of human oocytes and developmental potential and outcome after in vitro fertilization and embryo transfer. Hum. Reprod. 10, 415-24.

Wanka, F. \& Van Zoelen, E.J.J. (2003). Force generation by cellular motors. Cell. Mol. Biol. Lett. 8, 1017-33. 